\title{
Editorial About "More than 10-year Follow-up after Total En Bloc Spondylectomy for Spinal Tumors" (doi: 10.1245/s10434-013-3333-7)
}

\author{
Jean-Paul Wolinsky, MD and Ziya L. Gokaslan, MD \\ Department of Neurosurgery, Johns Hopkins University, Baltimore, MD
}

Kato et al. report 10-year follow-up data using the SF-36 on patients who underwent a spondylectomy. The authors' report is particularly useful because they collected the data on these patients with a long follow-up period. They discuss the pathology, outcome, complications, and patient satisfaction in this group of patients. The criteria for inclusion in this study was that a patient had to have undergone a spondylectomy and had to have survived at least 10 years from the time of the operation.

The title of the article- "More than 10-year Follow-up after Total En Bloc Spondylectomy for Spinal Tumors"implies that 10-year follow-up data will be presented on patients undergoing spondylectomy. The title is somewhat misleading: the outcome data reported is the follow-up data on patients who had undergone a spondylectomy and who had survived 10 years (29 patients). The data suggest that overall, these patients have done quite well with regard to their SF-36 scores. However, there is a significant bias. Patients who did not survive 10 years or patients who were lost to follow-up are not included in the data set. This means that there could be a significant selection bias. It may be that patients who do not survive 10 years have complications related to surgery or experience tumor progression. The SF-36 values in these patients could be quite poor, and analysis of these patients may suggest that they might not have benefitted from such a large operation. The opposite may be true as well. The data may show that although they did not survive 10 years, the operation was

(C) Society of Surgical Oncology 2014

First Received: 26 September 2013;

Published Online: 14 February 2014

J.-P. Wolinsky, MD

e-mail: jwolins3@jhmi.edu functionally beneficial to them, and the SF-36 outcome measures may be quite good. It would therefore be useful to include the other 53 patients out of the series of 82 patients, and to list the pathologies, survival times, complications, and SF-36 values at various time points including the last evaluation.

In addition, of the 29 patients included in this study, $35 \%$ did not undergo radiological follow-up at 10 years. The authors state that 23 of these 29 patients did not experience tumor recurrence at 10 years, but they also state that 10 of the 29 patients did not have radiological followup at 10 years. Even if all patients with radiological follow-up did not have a tumor recurrence, there are still four patients where there is no evidence to determine one way or another whether they experienced tumor recurrence.

Reviewing the patients who underwent a spondylectomy for a primary tumor, it is interesting to note the pathology of the patients who survived more than 10 years. Twentyeight patients underwent a spondylectomy for a primary tumor. Of these patients, 19 survived more than 10 years after surgery. No patients with a high-grade malignancy such as Ewing sarcoma, osteosarcoma, or malignant fibrous histiocytoma survived more than 10 years after the operation. Eleven of the 19 patients who survived more than 10 years had tumors that most likely would not have resulted in death, regardless of the surgical strategy (hemangioma, osteoblastoma, or giant cell tumor), and therefore the technique of a spondylectomy cannot really be credited with the patients survival. In addition, 1 of the 19 long-term survivors underwent a spondylectomy for a plasmacytoma; it can be argued that this patient underwent a far too aggressive surgical approach for a nonsurgical disease.

Dr. Tomita is one of the pioneers of the technique for the spondylectomy performed through an entirely posterior 
approach. This approach has been demonstrated to be useful in performing en bloc resections. A term coined by Tomita, the total en bloc spondylectomy (TES), has been used throughout the spinal literature, but the use of the term confuses the literature and makes literature reviews difficult to assess. A spondylectomy, by definition, is the removal of an entire segment of the spine; it gives no information about the margin of resection (intralesional or contaminated). An en bloc resection requires that the margin of the specimen is not violated. A total en bloc spondylectomy, although possible, is rarely, if ever, performed. It would require removal of the entire segment of the spine, in one piece, which would require sacrifice of the neural elements (spinal cord or cauda equina) within the spinal canal. The term total en bloc spondylectomy therefore is a misnomer and should not be used so as to avoid this confusion. Unfortunately, because of this confusion in terms, it is impossible to know what the true margins were for the various tumor pathologies treated. It would be useful to know if they were marginal en bloc or contaminated (planned transgression/intralesional) resections. Presenting this information would strengthen the article if the surgical margins of the patients who survived and who did not survive the 10 -year study period were clearly presented.

Despite the study's shortcomings, the authors' tremendous effort in collecting and providing information including a 10-year follow-up on patients who had undergone spondylectomy allows us to understand more about the treatment of primary tumors of the mobile spine. 